\title{
Analysis of Protocol Operations and Scalability of COPS-SLS Negotiation System
}

\author{
Thi Mai Trang Nguyen ${ }^{1,2}$, Nadia Boukhatem ${ }^{2}$, and Guy Pujolle ${ }^{1}$ \\ ${ }^{1}$ Laboratoire d'Informatique de Paris 6, 8 rue du Capitaine Scott, \\ 75015 Paris, France \\ \{Thi-Mai-Trang.Nguyen, Guy.Pujolle\}@lip6.fr \\ ${ }^{2}$ GET-Télécom Paris, LTCI-UMR 5141 CNRS, 46 rue Barrault, \\ 75013 Paris, France \\ \{trnguyen, boukhatem\} @enst. fr
}

\begin{abstract}
Dynamic service level negotiation is necessary to flexibly allocate resources according to QoS demands of a host or a domain. We have designed COPS-SLS as a service level negotiation protocol for IP-based networks. In this paper, we analyse a COPS-SLS-based negotiation system from the protocol design and system's scalability perspectives. This analysis is useful not only to the improvement of the COPS-SLS protocol design but also to the deployment of a service level negotiation system in general.
\end{abstract}

\section{Introduction}

To date, the relationship between customer and network as well as between network domains is based on static Service Level Agreement (SLA). The allocated network resources may be unused in low utilization periods or may be insufficient in high utilization periods. In addition, service levels in today's SLAs are offered only in one domain which is the domain of network provider. Once traffic is forwarded to another domain, the service level of the traffic is out of control. In the context of multiple domains, the offering of service levels is more complex because different domains can deploy different QoS architectures and apply different resource management policies. The motivation of our work is allowing SLA to be dynamically negotiated in order to enable the resource allocation on demand. Service levels can be negotiated and assured in any scope, one or multiple domains, specified by the customer, and independently of QoS architectures of individual domains while respecting the resource management policies of each domain.

We have defined the COPS-SLS (COPS usage for SLS negotiation) protocol for dynamic service level negotiation [1]. An SLS (Service Level Specification) can be considered as the technical part of the SLA which specifies the level of service that the traffic can obtain from the network. COPS-SLS extends the COPS protocol [2], which has been proposed for resource allocation in the context of Policy-based networking [3]. In this paper, we analyse a COPS-SLS based negotiation system from the protocol design and system's scalability perspectives. The organization of the 
remainder of this paper is as follows. Section 2 provides an overview on the COPSSLS protocol. The analysis of different operations of the COPS-SLS protocol impacting on the waiting time of a customer in a negotiation system is given in section 3. Section 4 presents a measurement of the scalability of a COPS-SLS negotiation system. Finally, section 5 concludes the paper.

\section{Protocol Overview}

We proposed COPS-SLS as an extension of the COPS protocol for the purpose of service level negotiation [1]. The SLS-PEP represents the customer which can be an end-host, a local network, an administrative domain, an ASP (Application Service Provider), or an ISP. The SLS-PDP represents the provider who provides service levels to customers. A provider can be an ASP or an ISP. SrNP (Service Negotiation Protocol) [5] and SIBBS (Simple Inter Bandwidth Broker Signaling) [6] are the protocols also designed for a similar purpose. SIBBS, SrNP and COPS-SLS are developed in a partially overlapping time periods during years 1999-2003. These three protocols have the common purpose of SLS negotiation, but each approach has its own particularities. The COPS-SLS protocol is organized in three phases as described in Fig. 1.

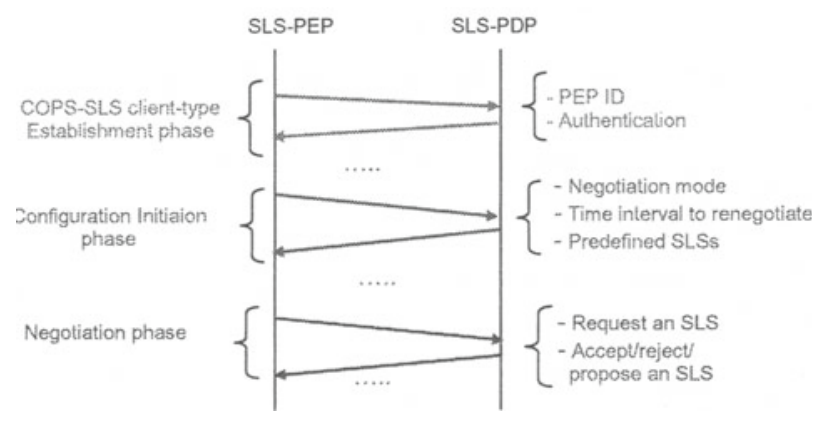

Fig. 1. COPS-SLS protocol

The system starts off with the COPS-SLS client-type establishment phase. The SLS-PEP must send its PEPID (PEP Identification) to the SLS-PDP for the authentication. If the SLS-PEP is authenticated, the COPS-SLS client-type is established and the system starts the configuration initiation phase. In this phase, the SLS-PEP and the SLS-PDP configure some parameters to prepare the negotiation process. Once the configuration of negotiation is successfully initiated, the system moves to the negotiation phase. In this phase, service levels can be negotiated and the configuration can also be changed if necessary.

In comparison with the alternative protocols such as SIBBS or SrNP, the configuration phase is the main difference distinguishing COPS-SLS from the others. This phase has been introduced in order for the negotiation to be able to flexibly adapt to a 
variety of customers, network providers, and QoS architectures. The customer and the provider are allowed to configure the manner of negotiation, and to change this configuration dynamically. The configuration parameters include the set of negotiation parameters, the negotiation mode, and the renegotiation interval. As different networks may provide guaranties of different QoS parameters and use different information to identify traffic, the agreement on these parameters allows the negotiation to be operational over heterogeneous environments. The set of negotiation parameters defines parameters which can be exchanged between the SLS-PEP and the SLS-PDP for the negotiation of a service level. For example, the SLS-PEP and the SLS-PDP can agree that only bandwidth and delay can be negotiated while the jitter parameter is not used in the negotiation. The negotiation mode defines how the customer can request a service level from the provider. COPS-SLS defines three negotiation modes and leaves the provider to choose the manner of negotiation which is most convenient to them. In the predefined-SLS mode, the provider advertises their customers of the service levels supported, using a set of predefined service levels. The customer can choose one of these predefined service levels to make a request. In the non-predefined $S L S$ with constraints mode, the provider does not supply their customers all the supported service levels but only put some constraints on the values of negotiation parameters sent in the requests. These constraints must be locally checked by the customer before sending a request. The non-predefined-SLS mode neither puts any constraint on the negotiation request nor pre-defines service levels. The customer asks the provider any desired service level. The renegotiation interval reflects the time interval before that a service level negotiated cannot be modified. Indeed, there is a trade-off between the negotiation's dynamic level and the network performance. The modification of a negotiated service level may lead to some network reconfiguration.

Another characteristic of COPS-SLS in comparison with the alternatives is that the SLS information exchanged between the PEP and the PDP is represented by a named data structure, a.k.a. a PIB (Policy Information Base) [4]. The advantage of using the concept of PIB is that the protocol becomes independent of the information carried.

\section{Analysis of Different Protocol Operations}

In this section, we analyse the impact of different operations of the COPS-SLS protocol on the starting time and the negotiation time. These times have an important influence on the customer's satisfaction. Large starting times or negotiation times may irritate the customers participating in the negotiation. The starting time is defined as the time taken by the system to be ready for the negotiation of a service level. Relating to the COPS-SLS design described in Fig. 1, this time includes the TCP connection time, the COPS-SLS client-type establishment time, and the configuration initiation time. The negotiation time is defined as the time taken by a customer to perform a negotiation of a service level in the negotiation phase.

To analyse the impacts on the starting time, the times taken by the following operations have been measured. The TCP connection time is measured between the time that the SLS-PEP tries to connect to the SLS-PDP and the time that the TCP connec- 
tion is established. The COPS-SLS client-type establishment time is measured between the time that the SLS-PEP sends an OPN (Client-Open) message (see [1] for COPS messages) to the SLS-PDP and the time that the SLS-PEP receives a CAT (Client-Accept) message from the SLS-PDP. The configuration initiation time is measured between the time that the SLS-PEP sends a $R E Q$ (Request) message with a 'context = configuration' to the SLS-PDP to initiate the configuration of negotiation and the time that the SLS-PEP finishes the transmission of a RPT (Report) message reporting a successful installation.

To analyze the impacts on the negotiation time, the following times have been measured. The negotiation time is measured between the time that the SLS-PEP sends a $R E Q$ message with a 'context = resource-allocation' to the SLS-PDP to negotiate a service level and the time that the SLS-PEP finishes the transmission of a RPT message reporting the final result of the negotiation. The decision time is measured between the time that the SLS-PDP receives a $R E Q$ message requesting a service level and the time that the SLS-PDP finishes making decision for this request.

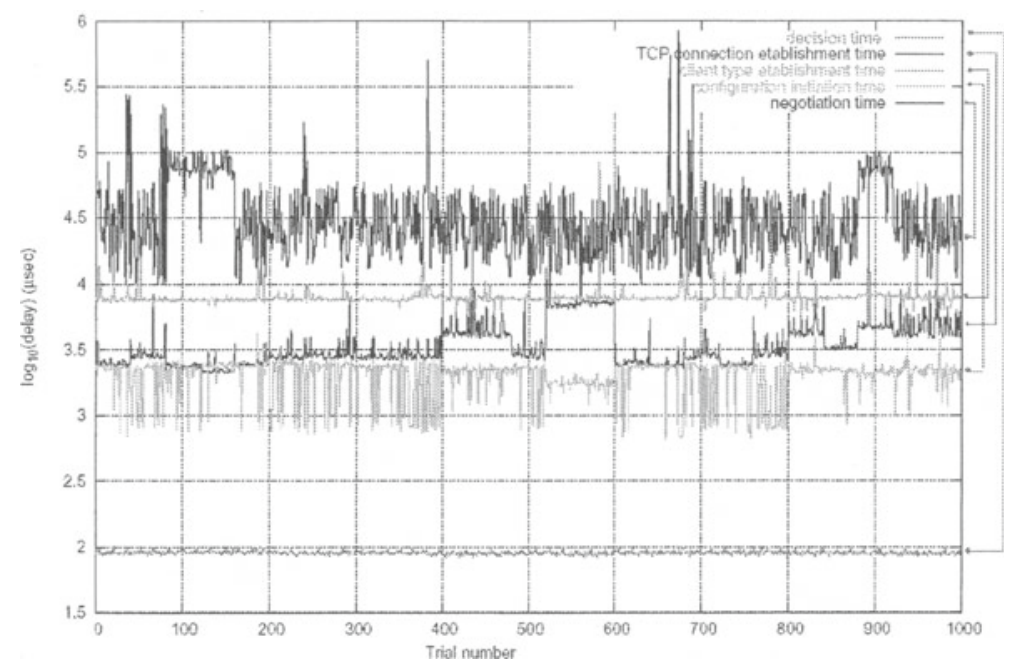

Fig. 2. Time measurements of different COPS-SLS protocol operations

One thousand trials have been realized from different SLS-PEPs to an SLS-PDP in a local network. In each trial, an SLS-PEP establishes the COPS-SLS connection with the SLS-PDP and realizes one negotiation. Fig. 2 presents the common logarithm of the times taken by the above-mentioned operations in each trial. The result shows that the operation that impacts most on the COPS-SLS starting time is the COPS-SLS client-type establishment, then the TCP connection establishment, and finally the configuration initiation. For the negotiation time, the time taken by the decision process of the SLS-PDP is minor in comparison with the transmission time of COPS-SLS messages. The analysis of this result leads to some conclusions on the design and the usage of a COPS-SLS based negotiation system. The COPS-SLS client-type estab- 
lishment is mandatory because this is an operation of any COPS-based protocol. The time taken by this operation can be minimized by reducing the time taken by the authentication process of the SLS-PDP. The TCP connection establishment is also an operation of any COPS-based protocol. However, different COPS client-types can use the same TCP connection. If the TCP connection is already established by another COPS client-type, the impact of the TCP connection time on the starting time can be eliminated. The configuration initiation time depends on the complexity of the configuration to be initiated. The configuration can be simple and minimized by designing a light PIB. The decision time depends on the decision process of the SLS-PDP. This time can vary with the volume of negotiation policies that the SLS-PDP handles, and the number of SLS-PEPs requesting service levels simultaneously. The distance between the SLS-PDP and SLS-PEPs and the message size should be taken into account for both the starting time and the negotiation time because of the transmission delay. The maximum message size in this test is around 300 bytes. The negotiation PIB should be carefully designed to minimize the volume of information encapsulated into a message.

\section{SLS-PDP Scalability}

This test aims at observing the behaviour of the negotiation system when the number of SLS-PEPs connecting to a SLS-PDP increases. In this scenario, 500 SLS-PEPs attempt to connect to only one SLS-PDP. The 500 SLS-PEPs are launched one by one every five seconds from 25 terminals (i.e. 20 SLS-PEPs are launched from a terminal) to the SLS-PDP in a local network. Once the COPS-SLS connection is established, each SLS-PEP negotiates service levels every one second.

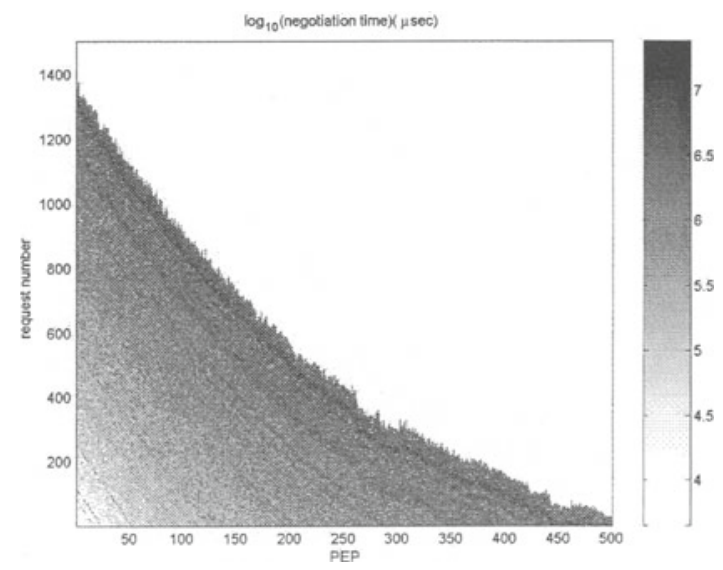

Fig. 3. Negotiation time measured in the scalability test of the negotiation system 
The common logarithm of the negotiation time measured in the system is presented in Fig. 3. The horizontal axis identifies the $i$ th SLS-PEP $(i=1: 500)$ connecting to the SLS-PDP. The vertical axis identifies the $k t h$ negotiation request that an SLSPEP can send to the SLS-PDP and obtain a decision. The colour represents the negotiation time of each request. This measurement shows that more the number of requests the SLS-PDP treats in a same lap of time, more the performance of the negotiation system is poor. When there are less than 50 SLS-PEPs in the system, the colour is light. The colour becomes darker as long as the number of connected SLSPEPs increases. When the system is up to 450 - 500 simultaneously connected SLSPEPs, the negotiation time can be up to $10^{6,5}$ and sometimes $10^{7}$ microseconds.

A negotiation system should be carefully dimensioned. The mechanism of redirecting a PEP to another PDP provided by the COPS protocol [4] sounds an interesting solution to the scalability of the negotiation system. If the number of SLS-PEPs connecting to an SLS-PDP reaches a threshold, the SLS-PDP can redirect further SLS-PEPs to another SLS-PDP. The re-negotiation interval adjustment is also a useful mechanism to put the negotiation rhythm under control.

\section{Conclusion}

In this paper, we summarized the main characteristics of COPS-SLS and analyzed a negotiation system from the protocol design and scalability perspectives. The analysis of different operations of COPS-SLS gives a concrete picture on the impacts on the starting time and the negotiation time. The scalability of the negotiation system has been studied at different number of simultaneous connected SLS-PEPs. The design of negotiation PIBs, the ability of simplifying or customizing the configuration initiation phase, and the dimensioning of a negotiation system are to be investigated in future works.

\section{References}

1. Nguyen, T.M.T., Boukhatem, N., Gharmri-Doudarn, Y., and Pujolle, G., COPS-SLS: A service level negotiation for the Internet. IEEE Communications Magazine, May 2002. 40(5): p. 158-165

2. Durham, D., et al., The COPS (Common Open Policy Service) Protocol. RFC 2748, January 2000

3. Yavatkar, R., D. Pendarakis, and R. Guerin, A Framework for Policy Based Admission Control. RFC 2753, January 2000

4. Chan, K., et al., COPS Usage for Policy Provisioning (COPS-PR). RFC 3084, March 2001

5. Goderis, D., et al., A service-centric IP quality of service architecture for next generation networks. in IEEE/IFIP Network Operations and Management Symposium. April 2002. Florence, Italy

6. Teitelbaum, B., et al., Internet2 QBone: Building a Testbed for Differentiated Services. IEEE Network, September/October 1999. 13(5): p. 8-16 